\title{
065
}

\section{RUOLO DEI BATTERI DEL GENERE \\ PSEUDOMONAS NELLE INFEZIONI OSPEDALIERE E TERRITORIALI}

Di Marcello V., Fabbrizi V.

Laboratorio Patologia clinica;

Ospedale Civile G. Mazzini Teramo Settore Microbiologia

Introduzione: I batteri del genere Pseudomonas sono bacilli gram negativi aerobi obbligati (tranne quelle specie che possono utilizzare la denitrificazione come mezzo do respirazione anaerobia).

Lo scopo del presente lavoro è di evidenziare il ruolo che $\mathrm{i}$ batteri del genere Pseudomonas hanno nella nostra realtà, il ruolo che essi svolgono nelle infezioni ospedaliere e ambulatoriali, lo stato dell'arte in materia di resistenze batteriche. Lo studio si è condotto in un periodo compreso tra il $1^{\circ}$ Gennaio 2001 e il 30 Giugno 2003. Si sono esaminati 14200 isolati.

Metodi: I campioni clinici presi in esame come significativi sono i seguenti: urine, espettorati, broncoaspirati, pus ferite, cateteri, liquidi cavitari ed emocolture. I materiali sono stati processati secondo protocollo e sulle eventuali crescite batteriche sono state eseguite identificazione ed antibiogramma con sistema automatizzato in micropiastra approvato NCCLS.

Risultati: Il 67\% dei batteri del genere pseudomonas isolati derivavano da pazienti ospedalizzati,il $33 \%$ da isolati di utenza esterna.

Le specie di pseudomonas isolate sono così distribuite:. 81\% Ps. Aeruginosa; 9\% St. Maltophilia; 8\% PS. Fluorescens/Putuda; 2\% B.Cepacia.

I reparti dai quali si ottengono il maggior numero di isolati per Pseudomonas, sono la Rianimazione generale e cardiochirurgia; la Chirurgia e la Pneumologia.

Caso a sé costituisce la Pediatra, essendo il nostro reparto centro regionale per il controllo della fibrosi cistica.

Non si sono rilevate differenze di pattern di resistenza, comparando il comportamento degli Pseudomonas isolati da campioni esterni o interni.Interessante è la particolare suscettibilità di questi batteri al trimetoprim/sulfametossazolo, farmaco efficace e poco costoso; fatta eccezione per lo Pseudomonas Aeruginosa, che mostra verso di esso resistenza costitutiva.

Conclusioni: Il diffuso e spesso ingiustificato uso degli antibiotici, contribuisce alla diffusione del fenomeno delle resistenze batteriche, un punto focale nella gestione delle infezioni ospedaliere e comunitarie.

Le reazioni del medico di base riguardo alle aspettative del paziente influenzano la pratica prescrittivi.Queste situazioni possono incoraggiare la selezione di ceppi resistenti, oltre che una spesa sanitaria non necessaria. 